\title{
Beyond the male sex hormone: deciphering the metabolic and vascular actions of testosterone
}

\author{
Hong-Yo Kang \\ Graduate Institute of Clinical Medical Sciences, Hormone Research Center, Kaohsiung Chang Gung Memorial \\ Hospital, Chang Gung University College of Medicine, Kaohsiung, Taiwan
}

Correspondence should be addressed to H-Y Kang

Email

hkang3@mail.cgu.edu.tw

\begin{abstract}
Among all the androgens that stimulate or control the development and maintenance of body composition, testosterone could be the most well known and important due to its linkage to many diseases, including the metabolic syndrome, type 2 diabetes, and cardiovascular disease. The detailed mechanisms of how testosterone functions in health and disease, however, remain unclear. During the past several decades, the successful cloning of the androgen receptor (AR) and its coregulators and establishment of AR transgenic and knockout animal models have led to rapid development in this field of study. The two thematic reviews in this issue of the Journal of Endocrinology provide a timely and useful guide and source of information to discuss the current knowledge of the metabolic and vascular actions of testosterone involvement in these androgen-related disorders. They described the mechanisms of relationships between testosterone and metabolic disease and how testosterone regulates vascular function and inflammation with a comprehensive summary of updated androgen-AR findings. As more research and clinical trials have put efforts into the study of how testosterone functions in these diseases, it is expected that the roles of testosterone and its actions will become clearer in the near future.
\end{abstract}
Key Words
> androgen
- androgen receptor
- cardiovascular
diabetes

Journal of Endocrinology (2013) 217, C1-C3
Testosterone - the primary form of androgen - is synthesized mainly in the gonads and secreted into the bloodstream where it can circulate and exert a number of diverse effects in peripheral target tissues at various locations. After entering the target cells but before it can perform its specific function by binding to the androgen receptor (AR), testosterone can either be metabolized into estradiol by aromatase or into dihydrotestosterone by $5 \alpha$-reductase. To maintain the appropriate concentrations of testosterone and its metabolites both in circulation and in the peripheral tissues, a dynamic network of multiple interacting factors involved in the cross talk between the androgen-targeted organs should be in perfect balance.
Persistent low serum testosterone levels in men with late-onset hypogonadism or androgen deficiency syndrome are associated with 'syndromic' disorders, including erectile dysfunction, low libido, decreased muscle mass and strength, increased fat mass, decreased bone mineral density, decreased erythropoiesis, decreased vitality, and depression. In addition, androgen deprivation therapy results in unfavorable changes in body composition, insulin resistance, and dyslipidemia and predisposes men to develop atherosclerosis and an increased risk of cardiovascular mortality. Thus, testosterone, the male gonadal hormone primarily known for its classical role in reproduction, becomes a hormone of metabolic and

Published by Bioscientifica Ltd. 
cardiovascular significance. However, our understanding of the mechanisms through which changes in testosterone might contribute to the health and disease of aging men remains rudimentary. The two thematic reviews in this issue of the Journal of Endocrinology provide a timely review of the evidence and discuss the current knowledge of the metabolic and vascular actions of testosterone in these disorders - specifically, how testosterone deficiency contributes to insulin resistance, metabolic syndrome, type 2 diabetes (Kelly \& Jones 2013a), and cardiovascular disease (Kelly \& Jones 2013b).

The hypogonadal-obesity cycle hypothesis was originally proposed by Cohen in 1999 to explain the relationship between low testosterone levels and metabolic disease. It was based on the finding that obesity impairs testosterone levels by increasing the aromatization of testosterone to estradiol, while low testosterone levels promote increased fat deposition (Cohen 1999). In their thematic review, Kelly \& Jones (2013a) have summarized the increasing evidence that adipocytokines contribute to low testosterone levels as well as to the processes underlying metabolic syndromes and type 2 diabetes; thus, they have extended on Cohen's theory to propose a hypogonadal-obesity-adipocytokine hypothesis. The presence of estradiol and the adipocytokines TNF- $\alpha$, IL6, and leptin (as a result of leptin resistance in obesity) inhibits the hypothalamic-pituitary-testicular axis response to decreasing androgen levels. This concept presented by Kelly \& Jones may explain why obesity impairs the negative feedback response of androgen production normally exerted by the hypothalamicpituitary-testicular axis when testosterone levels are low. Furthermore, the authors have reviewed the current literature on the contribution of testosterone deficiency to tissue-specific mechanisms involved in the development of obesity-induced insulin resistance, with special emphasis on testosterone-mediated glucose and lipid metabolism in the liver, muscle, and adipose tissue. An important point that can be derived from this publication is that changes in testosterone levels and metabolic profiling should be seriously considered. An increasing number of studies have illustrated the potential for applying metabolomics to the field of androgen research (Haring 2012). Thus, it becomes progressively more evident that system biology approaches, including the subsequent validation of candidate biomarkers using large-scale epidemiological data, will be of great value in improving the diagnosis and monitoring of androgen deprivation therapy in men.
Kelly \& Jones have also published another review article entitled, 'Testosterone: a vascular hormone in health and disease' in this issue of the Journal of Endocrinology (Kelly \& Jones 2013b). This review represents a new look at an old controversy. As early as the 1940s, the therapeutic use of testosterone was reported to improve angina pectoris in men with coronary artery disease (Lesser 1946). However, its therapeutic potential in cardiovascular medicine was generally overlooked, possibly due to the numerous case reports of sudden cardiovascular death among male athletes abusing anabolic steroids. Although long-term testosterone replacement appears to be a safe and effective means of treating hypogonadal elderly men (Hajjar et al. 1997), it was perceived earlier by the medical community to play a role in the development of cardiovascular disease and prostate cancer for many decades. The authors discuss the relationship between testosterone treatment and cardiovascular mortality in middle-aged and elderly men with low testosterone levels and point out that most of the epidemiological studies reported increased cardiovascular risk and mortality in men with low testosterone levels. They also provide evidence-based data on coronary blood flow and cardiac ischemia to confirm that the benefitto-risk ratio justifies both acute and chronic testosterone treatment. Notably, a recent interventional trial showed that testosterone treatment was associated with decreased mortality when compared with no testosterone treatment in an observational cohort of men with low testosterone levels (Shores et al. 2012). Although a number of shortterm studies conducted support the notion that testosterone therapy reduces the cardiovascular risk (Kelly \& Jones 2013b), a large long-term multicenter randomized trial will be required to prove whether testosterone therapy has a beneficial effect on cardiovascular risk and mortality. Such a trial should also follow patients for more than 5 years, use a combined measure of cardiovascular events and death as the primary outcome, and fully assess prostate safety and polycythemia.

The article also draws attention to the potential mechanisms of action of testosterone on vascular reactivity and inflammation. The majority of animal studies support the hypothesis that the actions of testosterone on vascular relaxation are both endothelium-dependent and independent vasodilatory effects. Endothelial-dependent actions of testosterone increase the expression or activity of endothelial nitric oxide synthase and enhance nitric oxide production, which in turn activates cyclic guanosine monophosphate to induce vasorelaxation in smooth muscle cells. Endothelial-independent mechanisms of

Published by Bioscientifica Ltd 
testosterone are believed to occur primarily via inhibition of voltage-operated $\mathrm{Ca}^{2+}$ channels and/or activation of $\mathrm{K}^{+}$channels in smooth muscle cells. Testosterone may also inhibit intracellular $\mathrm{Ca}^{2+}$ influx via store-operated $\mathrm{Ca}^{2+}$ channels by blocking the response to prostaglandin $\mathrm{F}_{2 \alpha}$. Although conflicting and contradictory experimental evidence exists, testosterone has demonstrated antiinflammatory effects to protect against atherogenesis in animal studies. Although the mechanisms of action of testosterone on vascular cells remain unclear, both genomic AR activation to modulate gene transcription and non-genomic activation to modulate the rapid intracellular signaling pathways of ion channels may mediate testosterone effects on vascular function and inflammation.

The complexity of the testosterone actions in health and disease is far beyond when Butenandt \& Ruzicka first showed how testosterone is synthesized and responsible for masculine characteristics in the early 1930s. There is substantial evidence on the role of testosterone in aging men with metabolic syndrome and cardiovascular disease which has completely changed its perception. It is now generally accepted that the testosterone action is multiple and that besides the well-known mainly reproductive functions, there are several metabolic and cardiovascular benefits, which are clearly summarized in these two review articles. Although examining the safety of testosterone treatment in aging men remains a concern, current studies shift the focus toward potential preventative strategies to help maintain androgens function in aging men. Recent work exploring the molecular structure and evolution of the AR in response to hormonal therapies has revealed novel mechanisms of AR-mediated diseases and yielded new targets for drug design. Future development of novel pro-anabolic pharmaceutical agents targeting the AR, which, together with other testosterone interventions, could allow testosterone to function properly for optimizing the management of metabolic syndrome and cardiovascular disease.

\section{Declaration of interest}

The author declares that there is no conflict of interest that could be perceived as prejudicing the impartiality of the research reported.

\section{Funding}

This research did not receive any specific grant from any funding agency in the public, commercial or not-for-profit sector.

\section{References}

Cohen PG 1999 The hypogonadal-obesity cycle: role of aromatase in modulating the testosterone-estradiol shunt - a major factor in the genesis of morbid obesity. Medical Hypotheses 52 49-51. (doi:10.1054/ mehy.1997.0624)

Hajjar RR, Kaiser FE \& Morley JE 1997 Outcomes of long-term testosterone replacement in older hypogonadal males: a retrospective analysis. Journal of Clinical Endocrinology and Metabolism 82 3793-3796. (doi:10.1210/jc.82.11.3793)

Haring R 2012 Perspectives for metabolomics in testosterone replacement therapy. Journal of Endocrinology 215 3-16. (doi:10.1530/JOE-12-0119)

Kelly DM \& Jones TH 2013a Testosterone: a metabolic hormone in health and disease. Journal of Endocrinology 217 R25-R45. (doi:10.1530/ JOE-12-0455)

Kelly DM \& Jones TH 2013b Testosterone: a vascular hormone in health and disease. Journal of Endocrinology 217 R47-R71. (doi:10.1530/ JOE-12-0582)

Lesser MA 1946 Testosterone propionate therapy in one hundred cases of angina pectoris. Journal of Clinical Endocrinology and Metabolism 6 549-557. (doi:10.1210/jcem-6-8-549)

Shores MM, Smith NL, Forsberg CW, Anawalt BD \& Matsumoto AM 2012 Testosterone treatment and mortality in men with low testosterone levels. Journal of Clinical Endocrinology and Metabolism 97 2050-2058. (doi:10.1210/jc.2011-2591)

Received in final form 10 February 2013

Accepted 25 March 2013

Accepted Preprint published online 25 March 2013
(C) 2013 Society for Endocrinology Printed in Great Britain
Published by Bioscientifica Ltd 